\title{
Auto-injection accidentelle d'épinéphrine par un bambin
}

\author{
Sarah Edgerley MD, Rongbo Zhu MD, Samira Jeimy MD PhD
}

— Citation : CMAJ 2021 April 26;193:E616. doi : 10.1503/cmaj.201798-f

Voir la version anglaise de l'article ici : www.cmaj.ca/lookup/doi/10.1503/cmaj.201798

$\mathbf{U}$

n garçonnet de 3 ans s'est présenté au service des urgences avec une douleur aiguë au genou gauche, incapable de porter son poids sur sa jambe gauche. Il souffrait d'une allergie aux arachides, pour laquelle on lui avait prescrit un auto-injecteur d'épinéphrine. Cinq jours auparavant, il avait sorti son auto-injecteur du sac à main de sa mère et s'était autoinjecté accidentellement le médicament dans le genou gauche avant que celle-ci puisse l'en empêcher.

L'examen a révélé une articulation chaude, enflée et érythémateuse avec des amplitudes de mouvement active et passive limitées. Une petite plaie punctiforme était visible sur la face latérale du genou, au point d'injection de l'épinéphrine (figure 1). Une radiographie a montré un important épanchement au niveau de la bourse suprapatellaire. L'analyse de laboratoire a révélé un nombre normal de globules blancs de 9,5 $\times 10^{9} / \mathrm{L}$ (plage normale $5,0-12,0 \times 10^{9} / \mathrm{L}$ ), un taux de protéine $\mathrm{C}$ réactive élevé de $81,6 \mathrm{mg} / \mathrm{L}$ (plage normale $0-5,0 \mathrm{mg} / \mathrm{L}$ ) et une vitesse de sédimentation érythrocytaire élevée de $97 \mathrm{~mm} / \mathrm{h}$ (plage normale $0-10 \mathrm{~mm} / \mathrm{h}$ ). Une arthrite septique a été diagnostiquée. Le patient a subi d'urgence une irrigation et un débridement de l'articulation sous anesthésie générale, permettant d'extraire environ $50 \mathrm{~mL}$ de matière purulente. Les cultures d'échantillons ont confirmé la présence de Staphylococcus aureus. Nous avons prescrit de la céfazoline pendant l'hospitalisation, puis de la céphalexine pendant 6 semaines. Après son congé de l'hôpital, le patient a continué à porter l'auto-injecteur d'épinéphrine.

Les auto-injecteurs d'épinéphrine intramusculaires constituent le traitement de première intention pour l'anaphylaxie ${ }^{1}$.

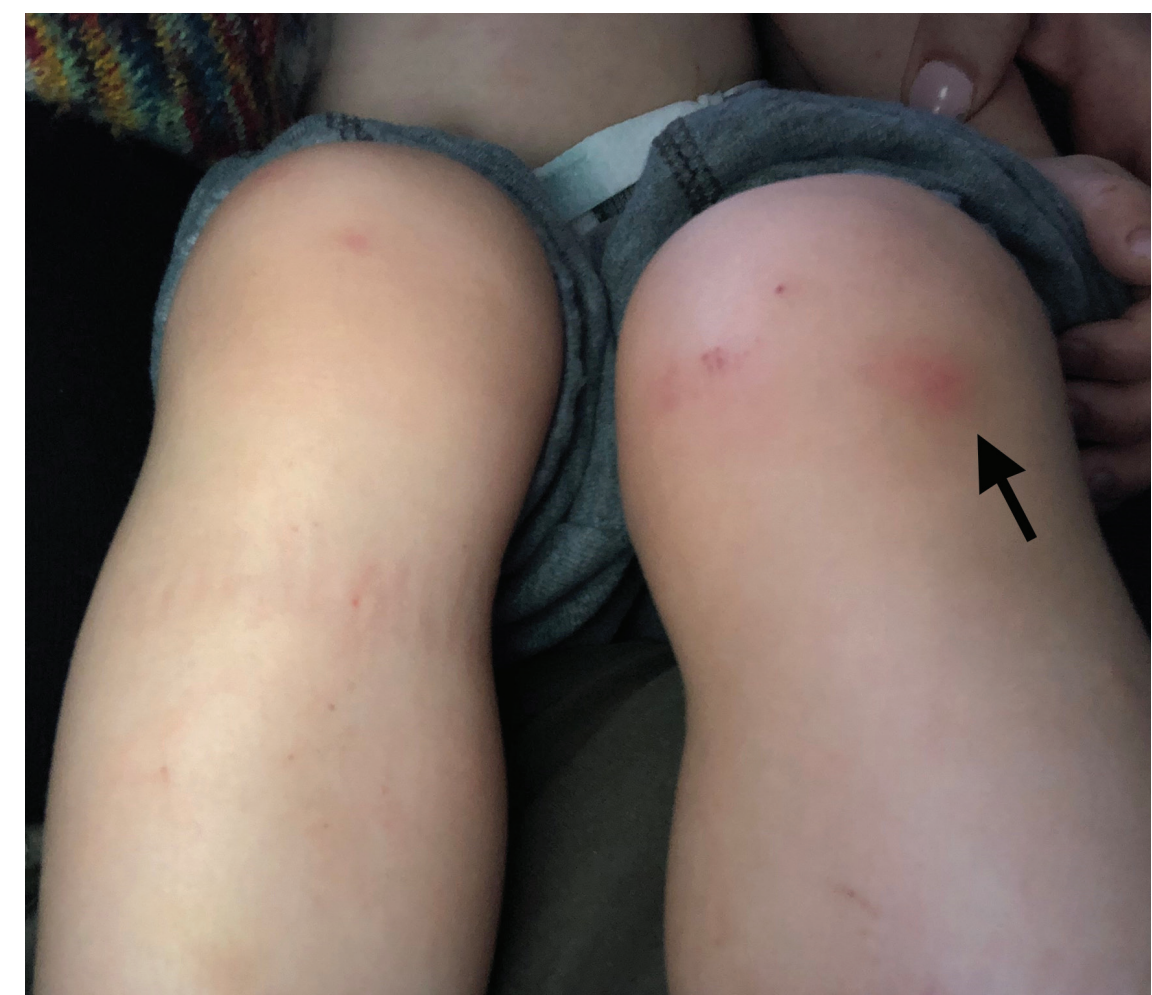

Figure 1 : Arthrite septique chez un garçonnet de 3 ans. Photo du genou gauche enflé du patient. On peut observer une petite zone d'érythème sur la face latérale du genou gauche au point de l'auto-injection accidentelle d'épinéphrine.

La hausse des taux d'allergies alimentaires a entraîné une augmentation des ordonnances d'auto-injecteurs d'épinéphrine ${ }^{1}$. L'administration accidentelle d'épinéphrine est une complication rare (une pour 50000 auto-injecteurs prescrits) ${ }^{2}$. Environ $45 \%$ des blessures sont subies par des enfants de moins de 11 ans $^{1}$. Les doigts sont les points d'injection accidentelle les plus courants qui entraînent des effets passagers et bénins ${ }^{1,2}$. Le traitement en est un de soutien, mais la phentolamine, la terbutaline et la nitroglycérine peuvent être utilisées en cas d'ischémie digitale². 
Les lignes directrices actuelles ne précisent pas d'âge minimum auquel les enfants peuvent s'auto-administrer de l'épinéphrine; cette décision doit être fondée sur le degré de maturité du patient et sa capacité à utiliser adéquatement l'auto-injecteur. Comme les bénéfices du port de l'auto-injecteur d'épinéphrine l'emportent largement sur les risques, il est recommandé d'inviter les patients à se tourner vers les ressources didactiques accessibles sur les sites Web des fabricants, et à se munir d'une trousse de formation et d'un plan d'action pour l'anaphylaxie ${ }^{3}$.

\section{Références}

1. Simons FER, Edwards ES, Read EJ, et al. Voluntarily reported unintentional injections from epinephrine auto injectors. J Allergy Clin Immunol 2010;125: 419-23.e4.

2. Mathez C, Favrat B, Staeger P. Management options for accidental injection of epinephrine from an autoinjector: a case report. J Med Case Rep 2009;3:7268.

3. Anaphylaxis emergency plan. Toronto: Food Allergy Canada. Accessible ici : https://foodallergycanada.ca/tools-and-downloads/downloads/emergency -plan-forms/ (consulté le 12 oct. 2020).
Intérêts concurrents : Samira Jeimy signale avoir participé aux services de conférenciers d'Aralez, d'AstraZeneca, de Sanofi, de Medexus, de Stallergenes Grier et de Novartis, et avoir siégé au conseil consultatif de Sanofi. Aucun autre intérêt concurrent n'a été déclaré.

Cet article a été révisé par des pairs.

Les auteurs ont obtenu le consentement des parents.

Affiliations : École de médecine et de dentisterie Schulich (Edgerley, Zhu, Jeimy), Division d'immunologie clinique et d'allergologie (Zhu, Jeimy), Département de médecine, Université Western, London, Ont.

Propriété intellectuelle du contenu : Il s'agit d'un article en libre accès distribué conformément aux modalités de la licence Creative Commons Attributions (CC BY-NC-ND 4.0), qui permet l'utilisation, la diffusion et la reproduction dans tout médium à la condition que la publication originale soit adéquatement citée, que l'utilisation se fasse à des fins non commerciales (c.-à-d. recherche ou éducation) et qu'aucune modification ni adaptation n'y soit apportée. Voir : https://creativecommons.org/licenses/by-nc-nd/4.0/deed.fr.

Correspondance : Samira Jeimy, samira.jeimy@lhsc.on.ca 